\title{
Arborescences
}

Revue d'études françaises

\section{Lettres d'Orient : échanges épistolaires en contexte interculturel dans les Mémoires du chevalier d'Arvieux}

\section{Vanezia Pârlea}

Numéro 9, décembre 2019

La lettre érudite. Nouvelles recherches sur la communication savante à l'époque moderne (XVI ${ }^{\mathrm{e}}$-XVIII ${ }^{\mathrm{e}}$ siècles)

URI : https://id.erudit.org/iderudit/1068275ar

DOI : https://doi.org/10.7202/1068275ar

Aller au sommaire du numéro

Éditeur(s)

Département d'études françaises, Université de Toronto

ISSN

1925-5357 (numérique)

Découvrir la revue

Citer cet article

Pârlea, V. (2019). Lettres d’Orient : échanges épistolaires en contexte interculturel dans les Mémoires du chevalier d'Arvieux. Arborescences, (9), 68-80. https://doi.org/10.7202/1068275ar

\section{Résumé de l'article}

$\mathrm{Au}$ XVII ${ }^{\mathrm{e}}$ siècle, la multiplication des voyages entraîne une consolidation des réseaux viatiques et épistolaires qui contribuent à l'avancement du savoir au sein de la République des Lettres. La correspondance entretenue par le chevalier d'Arvieux, consul d'Alep, avec l'évêque François Picquet, ambassadeur du Roi en Perse, et avec son aumônier Casmon entre 1681 et 1683 apparaît comme une illustration des échanges entre amateurs éclairés de l'Orient, dont l'étude sera une occasion d'analyser le fonctionnement d'un réseau viatique en contexte interculturel. Nous pourrons voir comment les lettres représentent la plupart du temps un agent de cohésion au sein de la communauté éparpillée et souvent démunie des voyageurs, en même temps porteurs d'un savoir et d'un goût de l'Ailleurs qu'ils sont seuls à pouvoir comprendre intimement. D'où toute une série de sous-entendus, de clins d'oeil disséminés à travers la correspondance, autant de marques de reconnaissance d'un savoir partagé et d'une érudition spécifique aux voyageurs d'Orient.
Tous droits réservés @ C Département d'études françaises, Université de Toronto, 2020
Cecument est protégé par la loi sur le droit d'auteur. L'utilisation des services d’Érudit (y compris la reproduction) est assujettie à sa politique d'utilisation que vous pouvez consulter en ligne.

https://apropos.erudit.org/fr/usagers/politique-dutilisation/ 


\section{SOMMAIRE}

1 Sébastien Drouin, Université de Toronto

Camelia Sararu, Université de Toronto

Introduction

10 Myron McShane, Centre d'études sur la Réforme et la Renaissance, Université de Toronto Une lettre de Jean Dorat sur l'œuvre de Nonnos

31 Benoît Autiquet, Université de Bâle

Ce que la lettre familière fait au discours médical.

Une lecture de la lettre XIX, 16 des Lettres de Pasquier (1619)

49 Guillaume Bazière, Université Paris Nanterre

Présence érudite et savoirs politiques dans la correspondance du Grand Condé

68 Vanezia Pârlea, Université de Bucarest

Lettres d'Orient : échanges épistolaires en contexte interculturel dans les Mémoires du chevalier d'Arvieux

81 Yves Moreau, Université de Lyon

"Qualche novità litteraria»: la correspondance

entre Jacob Spon (1647-1685) et Antonio Magliabechi (1633-1714)

95 Corinne Marchal, Université de Franche-Comté

La dynamique de la circulation des savoirs et de leurs matériaux dans les échanges épistolaires entre Jean-Baptiste Boisot et Paul Pellisson-Fontanier (1674-1693)

106 Mathilde Chollet, Le Mans Université

Un «gai savoir»: stratégies du rire dans les lettres

$d$ 'une érudite des Lumières 


\title{
Lettres d'Orient: échanges épistolaires en contexte interculturel dans les Mémoires du chevalier d'Arvieux
}

\author{
Vanezia Pârlea, Université de Bucarest
}

\section{Résumé}

Au XVII ${ }^{\mathrm{e}}$ siècle, la multiplication des voyages entraîne une consolidation des réseaux viatiques et épistolaires qui contribuent à l'avancement du savoir au sein de la République des Lettres. La correspondance entretenue par le chevalier d'Arvieux, consul d'Alep, avec l'évêque François Picquet, ambassadeur du Roi en Perse, et avec son aumônier Casmon entre 1681 et 1683 apparait comme une illustration des échanges entre amateurs éclairés de l'Orient, dont l'étude sera une occasion d'analyser le fonctionnement d'un réseau viatique en contexte interculturel. Nous pourrons voir comment les lettres représentent la plupart du temps un agent de cohésion au sein de la communauté éparpillée et souvent démunie des voyageurs, en même temps porteurs d'un savoir et d'un goût de l'Ailleurs qu'ils sont seuls à pouvoir comprendre intimement. D'où toute une série de sous-entendus, de clins d'œil disséminés à travers la correspondance, autant de marques de reconnaissance d'un savoir partagé et d'une érudition spécifique aux voyageurs d'Orient.

Dans l'Europe du XVII ${ }^{\mathrm{e}}$ siècle, le milieu intellectuel est directement concerné par les mutations en profondeur qui ont lieu à tous les niveaux, devenant le creuset où représentations et pratiques novatrices se développent graduellement. De nouvelles institutions apparaissent ou connaissent un nouvel essor, comme les Académies (Viala 1985), la presse ou le monde des livres (Schapira 2003), ce qui imprimera un dynamisme accru à la République des Lettres déjà consolidée. Dans ce contexte d' "une Europe de l'information" (Haffemayer 2008: 27), défini par une circulation des idées de plus en plus efficace, un rôle central revient à la correspondance, les nombreux acteurs des milieux savants se constituant en une communauté active, organisée sous forme de réseaux interpersonnels, où les nouvelles se répandent avec plus de vitesse qu'auparavant, sous l'effet, entre autres facteurs, du développement des services de la poste.

Cependant, ces réseaux érudits, qu'ils soient privés ou institutionnels, ne se bornent pas à faciliter la circulation des savoirs et leur diffusion à l'échelle européenne. Car, si l'on a affaire à une véritable culture de la mobilité (Beaurepaire et Hermant 2012), qui fleurit sur le vieux continent, celle-ci ne prend tout son sens qu'à un niveau extra-européen, à la faveur d'un développement sans précédent de la pratique du voyage et des types de discours corrélatifs. En effet, si le xvi ${ }^{\mathrm{e}}$ siècle est considéré comme l'époque des grandes découvertes géographiques, l'âge classique peut être envisagé sous l'angle d'une professionnalisation et d'une spécialisation croissantes du voyage.

Les protagonistes de ces échanges sont dès lors non seulement les savants professionnels, mais aussi les voyageurs infatigables, de plus en plus nombreux, qui, tout en arpentant le monde, restent en contact aussi bien avec leurs pairs, qu'ils rencontrent parfois en chemin, qu'avec l'élite intellectuelle. Certains voyageurs devenus fameux à l'époque, comme Jean Thévenot, Jean-Baptiste Tavernier ou François Bernier, sont considérés comme de véritables repères pour un public cultivé, de plus en plus fasciné par le Levant ou par les contrées de l'Asie éloignée. 
Dans ce contexte dynamique, les Mémoires du chevalier d'Arvieux s'avèrent un reflet fidèle des réseaux interpersonnels et épistolaires qui ont cours à l'époque. Situés à la croisée des genres, s'apparentant aussi bien au récit de voyage qu'au journal de négociation, genre diplomatique par excellence, ces volumineux Mémoires, dont les six tomes dépassent largement la longueur habituelle des relations de voyage, constituent un véritable carrefour (inter) culturel. On en veut pour preuve les innombrables expériences de l'Ailleurs dont le texte rend compte, qui ne mettent pas uniquement en scène l'auteurvoyageur ayant passé plus de vingt ans dans l'Empire ottoman, mais aussi d'autres personnages, dont la voix se fait parfois entendre directement à travers les lettres insérées à l'intérieur du récit.

En effet, l'un des mérites des Mémoires du chevalier d'Arvieux est de donner la parole à l'Autre, selon une pluralité de stratégies discursives à même d'apporter un nouvel éclairage sur les choses vues et les aventures vécues. Le voyageur-écrivain est ainsi bien plus enclin que d'autres à laisser s'affirmer des points de vue différents du sien, même si cela n'est pas toujours à son avantage. Le dernier tome des Mémoires en est un bon exemple. Il témoigne, entre autres choses, des déboires d'un ami de Laurent d'Arvieux, François Picquet, ancien consul d'Alep et missionnaire devenu ambassadeur du roi en Perse, à travers une série de lettres érudites de l'ancien consul et de son aumônier dont le ton familier laisse percer un extraordinaire sens du vécu. L'analyse de cette correspondance illustrera ce croisement de voix et de regards qui constitue l'une des spécificités des Mémoires du chevalier d'Arvieux, tout en reflétant également certaines particularités des réseaux épistolaires et viatiques sous l'Ancien Régime.

\section{Réseaux épistolaires et viatiques}

Parmi les types de voyage dont l'essor est soutenu par la politique de l'État colbertiste, qui finance des missions culturelles et scientifiques, l'un des plus prisés est le voyage savant et érudit, en particulier sa spécialisation «antiquaire». Cependant, loin de se présenter à l'état pur, il reste plutôt un idéal; il entend concilier l'esprit scientifique et érudit avec le souci classique d'instruire et de plaire et, ce faisant, joindre l'utile à l'agréable. C'est ainsi que

[...] le récit savant n'est presque jamais un récit spécialisé; c'est, au contraire, un type de récit de voyage qui, tout en recherchant un but spécifique, voudrait profiter de l'occasion pour donner toutes sortes d'informations et pour animer autant que possible le côté professionnel qui risquerait d'ennuyer le lecteur moyen. [...] Voyager en savant, c'est faire preuve d'une vaste érudition dans plusieurs domaines, posséder à fond les langues des pays parcourus [...] Ce type de récit semble donc répondre aux exigences d'un public cultivé comme à l'idéal de la République des Lettres [...]. (Wolfzettel 1996: 191-192)

La destination privilégiée de ces missions, entreprises souvent "par ordre du Roi» et censées la plupart du temps enrichir le cabinet de curiosités de ce dernier ou la bibliothèque royale, reste l'Orient ${ }^{1}$. C'est d'ailleurs à cette époque que l'intérêt des érudits envers les langues orientales, et plus généralement envers ces contrées éloignées, amène la constitution d'une première forme d'orientalisme «baroque» ou «early modern Orientalism» (Dew 2009: 1-40). En effet,

[1] es études orientalistes, suivant en cela l'évolution des autres disciplines, se spécialisent et se professionnalisent pendant le XVII ${ }^{\mathrm{e}}$ siècle, avec la création de chaires d'arabe dans les principaux centres

1. Le terme «Orient» reste encore bien flou à l'époque et peut désigner tantôt le Levant, assimilé surtout à l'Empire ottoman, tantôt des contrées plus éloignées, comme la Perse safavide ou l'Inde moghole. 
d'enseignement et de recherche européens. [...] Les études sur l'Orient sont alors essentiellement liées à l'apprentissage et à la maîtrise des langues. (Heyberger 2015: 507)

Il s'agit d'un réseau de savants qui entreprennent des recherches sur l'Orient, à l'instar de Barthélemy d'Herbelot ou de Melchisédech Thévenot, mais qui sont également engagés dans des réseaux épistolaires et interpersonnels aussi bien avec leurs pairs qu'avec les vrais témoins que sont les voyageurs. On assiste ainsi à la constitution et au développement d'une véritable «République des lettres orientaliste» (Girard 2015: 387), dont les travaux ne restent pas sans écho parmi les représentants de tout un public lettré, curieux et dont l'intérêt envers l'Islam va croissant.

Il arrive aussi que de jeunes érudits entreprennent eux-mêmes des voyages au Levant pour en ramener médailles, monnaies, manuscrits, informations, etc. Parmi les figures importantes pour l'espace français, citons Antoine Galland, le célèbre orientaliste et futur traducteur des Mille et une nuits qui accompagna lors d'un premier voyage à Constantinople le marquis de Nointel, ambassadeur de France à la Porte, en tant que secrétaire personnel, ou encore François Bernier, voyageur philosophe disciple de Gassendi, célèbre pour son long séjour dans l'Inde Moghole et pour les lettres savantes qui en rendent compte. Ainsi, qu'il s'agisse d'érudits de cabinet, de voyageurs savants ou tout simplement curieux, ceux-ci forment, malgré les tensions et les rivalités qui les animent parfois, une communauté d'intérêts et d'affinités unie à travers des réseaux épistolaires et viatiques.

À l'image du récit de voyage savant, la lettre érudite prend dans ce contexte d'exploration de l'Orient les traits de ce «genre sans loi » qu'est le genre viatique auquel elle s'apparente (Le Huenen 1990: 14), à savoir son intertextualité, son hétérogénéité, en alliant érudition encyclopédique et ton mondain, voire badin. Étant donné que ce genre évolue d'une circulation manuscrite, à usage privé, vers une publication à plus grande échelle, le souci du public auquel on s'adresse devient lui aussi un élément important qui, à plusieurs reprises, infléchit le discours vers le côté divertissant et savoureux de la découverte de l'Ailleurs, à même de séduire non seulement les savants, mais aussi un lectorat plus large, formé d'esprits instruits et curieux. Un certain nombre de voyageurs, qui n’appartiennent pas véritablement à la catégorie des voyageurs savants et sont animés principalement par d'autres buts ou motivations, qu'ils soient commerciaux, diplomatiques ou encore religieux, peuvent ainsi être vus comme des amateurs éclairés de "choses» en provenance d'Orient.

Ce fut le cas de Laurent d'Arvieux et de François Picquet, ces deux voyageurs qui entretinrent, à la faveur de leur amitié, une correspondance des plus intéressantes. L'étude de ces échanges épistolaires sera l'occasion d'analyser la construction d'un réseau viatique et son fonctionnement en contexte interculturel à l'âge classique.

\section{Amitié, voyage et correspondance}

À travers ses longs séjours dans l'Empire ottoman, le chevalier d'Arvieux ${ }^{2}$ évolue: le jeune commerçant féru de langues orientales devient peu à peu un diplomate accompli et bien inséré dans toute

2. Laurent d'Arvieux (1635-1702), plus connu sous le nom du chevalier d'Arvieux, est un voyageur français du $\mathrm{XVII}^{\mathrm{e}}$ siècle, l'un des premiers à avoir séjourné dans un camp d'Arabes nomades, chez les Bédouins du mont Carmel (1664-1665). Après un premier séjour de douze ans dans l'Empire ottoman (1653-1665) où il s'initie au commerce, le chevalier d'Arvieux rentre en France et sera chargé de plusieurs missions diplomatiques, étant nommé envoyé à Tunis, envoyé extraordinaire du roi à la Porte, consul de France à Alger et, finalement, consul de France à Alep. En 1669, il sera également désigné en tant qu'interprète officiel du roi lors de la fameuse ambassade de Soliman Aga, 
une série de réseaux (inter) culturels de la seconde moitié du Xvir ${ }^{\mathrm{e}}$ siècle. Ses $M e ́ m o i r e s{ }^{3}$, dont la seule édition complète paraît après sa mort, en 1735, rendent compte de ses voyages et missions en Orient et relèvent de la relation de voyage, genre hybride par excellence. Alternant entre la narration chronologique, les descriptions et des digressions où l'anecdotique côtoie l'érudition, les six tomes renferment également des documents de toutes sortes, y compris des lettres. Les dernières fonctions diplomatiques que d'Arvieux exercera entre 1679 et 1686 seront celles de consul d'Alep, fonctions dont il s'acquittera honorablement, malgré toute une série de difficultés dont il rendra compte dans le sixième et dernier tome de ses Mémoires. C'est au mois de septembre 1679 qu'il s'embarque à Toulon en compagnie de François Picquet, lui-même ancien consul d'Alep pendant neuf ans, qu'il connaissait déjà depuis longtemps, d'après son propre aveu ${ }^{4}$.

La trajectoire professionnelle et personnelle de son illustre prédécesseur avait été jusqu’alors des plus insolites ${ }^{5}$, lui qui incarnait la figure singulière du diplomate-missionnaire. À ce moment-là, ce dernier reprenait le chemin de l'Orient en sa nouvelle qualité d'évêque de Césarople et de Babylone, avant d'exercer, par la suite, les fonctions d'ambassadeur du roi en Perse. C'est le 30 avril 1681 que François Picquet commence son voyage vers la Perse dont il ne reviendra jamais ${ }^{6}$. Ce départ marque également le début d'une correspondance soutenue entre l'ancien et le nouveau consul d'Alep, qui s'étendra de 1681 jusqu'en 1683. Le dernier tome des Mémoires du chevalier d'Arvieux rassemble en tout une quinzaine de lettres de François Picquet ${ }^{7}$, ainsi que quatre lettres de son aumônier, M. Casmon, décédé à

l'envoyé du Sultan à Paris. Par la suite, le roi lui-même lui demandera de collaborer avec Molière et Lully pour la pièce Le bourgeois gentilhomme. Pour plus de détails concernant son parcours oriental, voir Pârlea (2015) et Lewis (1962).

3. La seule version complète des Mémoires de Laurent d'Arvieux sera publiée de manière posthume en 1735 par le dominicain Jean-Baptiste Labat et comporte toute une série d'interventions et de remaniements de la part de l'éditeur. Une édition antérieure datant de 1717, celle de Jean de la Roque, ne contient que le récit de son séjour chez les Bédouins du mont Carmel et une présentation détaillée de leurs mœurs et coutumes. Il nous reste, en outre, un manuscrit non autographe incomplet, conservé à la bibliothèque de Versailles, connu sous le nom du manuscrit Lebaudy; le dernier tome, celui qui devait recouvrir la période de son consulat d'Alep, en est absent. Pour plus de détails, voir notre chapitre "Les Mémoires du chevalier d'Arvieux: une aventure seconde» (Pârlea 2015: 41-50) et Hossain $(1990 ; 1997)$.

4. "J'étois ami de ce Prélat depuis long-tems» (d'Arvieux, Mémoires V: 424).

5. François Picquet (1626-1685) est un diplomate et un ecclésiastique français du Xvir e siècle. Né à Lyon, dans «[...] une famille aussi considerable par sa noblesse, que par ses biens et par sa pieté» (d'Arvieux, Mémoires VI: 81-82), François Picquet eut dès sa jeunesse une inclination particulière pour l'état ecclésiastique, mais, pour des raisons familiales, il dut d'abord suivre une carrière commerciale et diplomatique. Nommé consul de France à Alep, il exercera cette fonction entre 1652 et 1661 et s'affirmera comme un véritable protecteur des missionnaires catholiques en Orient. De retour en Europe, il entre dans les ordres et se voit octroyé le prieuré de Grimaud, en Provence. Tenu en haute estime pour sa piété et son dévouement à la cause religieuse, il sera sollicité pour de nouvelles missions en Orient, étant nommé successivement vicaire apostolique de Babylone, évêque in partibus de Césarople, ainsi qu’ambassadeur du roi en Perse. Son statut de diplomate et d'ecclésiastique, à première vue contradictoire, est également mis en évidence par Charles Léonce Octavien Antelmy, auquel on attribue une biographie de l'évêque: «Monsieur Picquet ne réünissoit pas seulement en lui des fonctions qui ne paroissoient pas compatibles, mais sa douceur, sa modestie et sa modération dans les sentimens et dans la conduite, le rendoient cher à toutes les personnes, qui de son tems aimoient l'Eglise" (Antelmy, La vie de messire François Picquet: iv-v). Sur la vie et l'activité de François Picquet, voir aussi Zeiller (1943) et El-Mudarris et Salmon (2009).

6. Il s'éteint le 26 août 1685 à Hamadan, en Perse.

7. Selon les précisions qui figurent en manchette, certaines de ses lettres ont malheureusement été perdues. Les lettres de d'Arvieux n'ont pas été incluses dans les Mémoires. 
Ispahan en $1682^{8}$. Nous avons affaire à des épîtres croisées, ce qui explique le regard double porté parfois sur les mêmes événements. Ces lettres sont entremêlées au récit de l'activité consulaire de d'Arvieux selon la formule digressive du genre viatique, parmi d'autres textes et documents, dont une description de la ville d'Alep jugée comme la plus intéressante et la plus complète de son temps.

La ville d'Alep revêtait une grande importance aux yeux de la monarchie française. Troisième ville de l'Empire ottoman en grandeur et en importance, Alep avait depuis longtemps une position privilégiée, voire stratégique, étant située sur les principales routes commerciales terrestres. En tant que ville carrefour entre Orient et Occident, elle représentait un nœud commercial et culturel et était réputée pour sa grande diversité ethnique et confessionnelle, pour la beauté de ses caravansérails, ainsi que pour son commerce florissant de tissus précieux, en particulier celui de la soie. L'essor économique que la ville avait enregistré au XVI ${ }^{\mathrm{e}}$ siècle et au début $\mathrm{du} \mathrm{XVII}^{\mathrm{e}}$ siècle avait favorisé l'installation précoce des Vénitiens, suivis par des marchands français, anglais et néerlandais. La prospérité d'Alep sera remarquée par nombre de voyageurs français de l'âge classique, à l'image de Jean-Baptiste Tavernier:

Alep est une des plus celebres villes de la Turquie, tant pour sa grandeur et sa beauté, que pour la bonté de son air accompagnée de l'abondance de toutes choses, et pour le grand commerce qui s'y fait par toutes les nations du monde qui y abordent. (Tavernier, Les Six Voyages I: 134)

Du temps du consulat de Laurent d'Arvieux, la ville d'Alep était devenue encore plus importante en raison de "la politique orientale de Louis XIV et de son ministre Colbert, avec l'envoi de savants à la recherche de manuscrits et le renouvellement des capitulations par l'ambassadeur le marquis de Nointel (1674)»(Salmon 2011: 153). Dans ce contexte, le renouvellement des capitulations avait représenté un moment de réconciliation symbolique entre la France et l'Empire ottoman après une période de tensions diplomatiques. Il avait notamment permis aux Français de renforcer leur présence économique, religieuse et politique dans la région, tout en nourrissant leur volonté de domination dans la compétition qui les opposait aux autres nations européennes.

La monarchie française cherchait ainsi à étendre son influence du côté de l'Asie, essayant en cela de combler son retard par rapport aux autres pays européens, notamment les Anglais et les Hollandais, dans le commerce méditerranéen et asiatique. La création de la Compagnie des Indes orientales en 1664 témoignait déjà du nouveau positionnement stratégique de la France, qui se tournait enfin vers cet Orient lointain. Dans ce nouveau contexte géopolitique, Alep jouait un rôle d'autant plus important qu' elle constituait un nœud de communication où se formaient les caravanes en partance pour la Perse safavide et l'Inde moghole.

À la différence d'autres relations de voyage, comme par exemple celles de ses devanciers JeanBaptiste Tavernier ou François de La Boullaye-Le Gouz, pour lesquels la ville d'Alep n'est qu'un lieu de passage vers leurs véritables destinations, à savoir la Perse et l'Inde, le récit du chevalier d'Arvieux apparaît comme l'un des rares témoignages d'un voyageur résidant dans cet endroit d'une importance stratégique pour la Couronne. Ce faisant, il est particulièrement à même de rendre compte du détail des diverses missions françaises dans la région, qu'elles soient diplomatiques, culturelles ou religieuses. En même temps, en intégrant les «lettres persanes» de François Picquet et de son aumônier Casmon, les Mémoires de d'Arvieux font figure d'œuvre-carrefour, dont la polyphonie reflète mieux que d'autres ouvrages contemporains du même type le fonctionnement des réseaux épistolaires dans un contexte interculturel.

8. Information qui figure dans la lettre du 16 septembre 1682 adressée par François Picquet à Laurent d'Arvieux. 
La correspondance est précédée d'un bref chapitre introductif, intitulé «Histoire de M. François Picquet Evêque de Cesarople, Vicaire Apostolique de Babylone et Visiteur General de la part de sa Sainteté en Orient», dans lequel d'Arvieux retrace le parcours édifiant du saint homme. Voici de quelle manière le consul d'Alep entend justifier la nécessité d'un tel préambule:

Mais avant de rapporter les Lettres que j'ai reçûës de ce grand Prélat, qui ne seront pas indifferentes au public; je crois devoir à l'amitié dont il m’a honoré ce que j'ai pû ramasser de son histoire; si je ne le faisois pas, je croirois priver le public d'une chose qui lui fera plaisir. (d’Arvieux, Mémoires VI : 81)

Cette inclusion de lettres privées, précédées de l'histoire de l'illustre personnage, dans le circuit de la publication s'explique par une double motivation. D'une part, c'est l'amitié particulière ayant uni les deux voyageurs et diplomates, bâtie sur des affinités électives, qui apparaît comme un facteur déterminant. Le lien d'amitié est d'ailleurs pleinement confirmé à travers nombre de formules explicites de la part de son correspondant: "Je vous embrasse, Monsieur, comme mon bon et plus cher ami [...]» (d'Arvieux, Mémoires VI: 103); "Je suis ravi de votre confirmation pour trois ans dans le Consulat: il falloit mettre trente au lieu de trois et ne me pas marquer l'affoiblissement de vos yeux et de vos nerfs ${ }^{9}$; car j'en ai une veritable douleur, telle que la doit avoir un ami tendre et sincere [...]" (d'Arvieux, Mémoires VI: 157). Malgré le ton quelque peu convenu de ces phrases, l'affection et la compassion qu'elles expriment sont indéniables. Cette impression est encore renforcée par des formules qui, moins directes, n'en sont pas moins une preuve de la familiarité qui règne entre les deux correspondants: "Je vous écris sans ceremonie, faites-en de même et donnez-moi de vos nouvelles. Je souhaite apprendre que vous êtes content et en bonne santé» (d'Arvieux, Mémoires VI: 125); «[...] je vous ai fait le détail d'une partie de nos chagrins, qui excitera la compassion d'un cœur aussi tendre que le vôtre» (d'Arvieux, Mémoires VI: 95). C'est par le biais de sa correspondance que nous avons accès à un aspect inattendu de la personnalité du chevalier d'Arvieux, connu pour être un voyageur intrépide et curieux, mais pas particulièrement sentimental ${ }^{10}$, l'évocation de son "cœur tendre» ne pouvant se retrouver que sous la plume d'un ami intime. Ces quelques expressions de l'évêque nous font entrevoir l'existence d'une communion affective, fondée sur une complicité et une compréhension mutuelle, l'interlocuteur étant envisagé comme un alter ego.

À côté du lien affectif, le souci du public constitue l'autre motivation centrale pour la publication. En effet, les termes employés au sujet des lettres - «qui ne seront pas indifferentes au public» - ainsi que de la présentation abrégée de la vie de François Picquet - «je croirois priver le public d'une chose qui lui fera plaisir» - témoignent du fait que l'auteur était conscient de l'opportunité que représentait une telle publication. La justification proposée relève de cette même alliance entre l'utile et l'agréable,

9. Son éditeur, le père Labat, nous apprend en effet que "[l]e Chevalier d'Arvieux étoit attaqué depuis long-tems d'une fluxion sur les yeux, et d'un tremblement extraordinaire dans les bras et dans les mains [...]» (d'Arvieux, Mémoires VI: 482).

10. En effet, le genre viatique fait peu de place aux marques d'affectivité et à l'épanchement sentimental. François Moureau a bien raison de préciser en ce sens que «[l]e voyageur de l'âge classique est le plus souvent le grand absent de sa relation, même quand il s'exprime sur le mode personnel. [...] Ses angoisses, ses désirs, sa lassitude, sa nostalgie du pays natal? De tels sentiments n'affleurent même pas dans la plupart des récits» (Moureau 2005: 18). Il y a cependant des exceptions notables, comme par exemple les relations de voyage de Jean Chardin, bien plus enclin que d'autres à la confession et à l'expression de son ressenti. Pour plus de détails sur ces questions, voir Antoine et Pârlea (2018). 
mais trahit également la nature du public visé, curieux et cultivé, dont l'intérêt et le plaisir sont anticipés par d'Arvieux. Le portrait de l'évêque est, en effet, extrêmement élogieux:

[...] il avoit de l'esprit infiniment, il avoit appris en perfection les Langues Orientales, il avoit beaucoup étudié et connoissoit mieux que personne les interêts de sa Nation dans le commerce, et le genie des Turcs. (d'Arvieux, Mémoires VI: 82)

À côté de son profond attachement et de son soutien actif aux progrès des missionnaires et de la foi catholique, soutien évident chez d'Arvieux tout comme dans les autres biographies de François Picquet, les éléments importants qui figurent ici sont la connaissance approfondie des langues orientales, des affaires commerciales et des enjeux, pourrions-nous dire, interculturels. On voit alors s'esquisser non seulement le portrait d'un diplomate-missionnaire, mais aussi celui d'un érudit, possédant le savoir spécifique d'un voyageur en Orient et appartenant à la même communauté savante que son interlocuteur.

Cette correspondance nous montre ainsi un enchevêtrement de réseaux sociaux, (inter) culturels et épistolaires dans lesquels les deux correspondants sont insérés. Grâce à une mise en abyme opérée par la reprise des lettres de l'évêque, le public a accès au miroitement de tout un réseau épistolaire auquel Picquet appartient, celui-ci évoquant çà et là des lettres envoyées, reçues ou attendues avec impatience tantôt en provenance de Rome, tantôt de l'ambassadeur de Constantinople ou même des Indes. Arrivé à Diyarbakir ${ }^{11}$, l'évêque a l'occasion de rencontrer Mar Joseph, le patriarche des Chaldéens ${ }^{12}$, qu'il ne se fait pas faute de porter aux nues:

[...] les Turcs même le respectent, c'est beaucoup dire. Vous sçavez que je le connoissois depuis long-tems de reputation, et par les Lettres qu'il m’a écrites de Rome, où il a demeuré quelque tems, et d'autres endroits; j'ai été ravi de le connoître personnellement et de l'embrasser. (d'Arvieux, Mémoires VI: 98)

Ou alors dans une lettre du 4 juin 1681:

J'ai été jusqu'aujourd'hui dans l'esperance de recevoir de vos nouvelles avant mon départ: je comptois aussi que mes Procureurs m’envoyeroient des Lettres de Chrétienté par un messager exprès, mes esperances s'évanouïssent. [...] Je mets ici deux Lettres pour Rome par duplicata. Faites-moi la grace, Monsieur, de les envoyer par deux voyes differentes et avec sureté, je vous en serai bien obligé. (d'Arvieux, Mémoires VI : 101)

Ce ne sont que deux exemples parmi d'autres qui rendent compte de la complexité des réseaux aussi bien sociaux qu'épistolaires auxquels les deux Français appartiennent. Ils renvoient ainsi à «la diversification et à l'imbrication complexe des réseaux qui définissent l'espace social à l'âge classique (Häseler et McKenna 2006: 8), d'autant plus que les deux acteurs évoluent dans un contexte interculturel. Dans le premier passage, il s'agit d'une correspondance de longue date entre représentants de l'Église que seul le voyage peut réunir en chair et en os. Dans le deuxième cas, nous voyons le chevalier

11. Ville de l'actuelle Turquie que se disputent au XviI ${ }^{\mathrm{e}}$ siècle la Sublime Porte, autrement dit l'Empire ottoman, et la Perse safavide.

12. Mar Joseph I, patriarche de l'Église chaldéenne (1681-1696), l'une des Églises catholiques orientales de tradition syriaque, auparavant nestorienne. 
d'Arvieux jouer un double rôle, faisant figure tantôt d'interlocuteur privilégié, d'ami et de confident auquel l'évêque confesse ses "espérances» et ses inquiétudes, tantôt d'intermédiaire expérimenté et dévoué, prêt à rendre service à tout instant, particulièrement soucieux des aléas des routes et de la poste en pays étranger. Les deux hommes constituent non seulement des maillons au sein de leurs réseaux épistolaires respectifs, mais aussi au sein d'un réseau plus large servant chacun de relais et participant à la construction de leur identité sociale.

En effet, la figure du voyageur érudit est représentée non seulement par les destinateurs (Picquet et, dans une certaine mesure, Casmon), mais également, d'une manière plus subtile, par le destinataire. Les indices abondent en ce sens à travers la correspondance. En évoquant les festins qui, une fois arrivés à Diyarbakir, lui sont offerts à lui et à ses gens par Mar Joseph, le patriarche des Chaldéens, Picquet précise la chose suivante: "A tout autre qu’à vous je ferois le détail de ces festins, mais je suis sûr que vous en sçavez plus que je ne pourrois vous en dire» (d'Arvieux, Mémoires VI: 99). De son côté, l'aumônier Casmon écrira, dans une lettre du 28 janvier 1682 pleine de déférence, dans laquelle il raconte leur traversée périlleuse des montagnes vers la Perse entre Diyarbakir et Erzeroum ${ }^{13}$ :

Vous voyez bien, Monsieur, que je vous parle de la richesse des simples ${ }^{14}$, que les curieux comme vous eussent regardé avec plaisir et une connoissance parfaite; pour moi je vous assure que j'eusse mieux aimé un bon potage [...] Nous eumes pourtant l'honneur de passer fort fierement l'endroit fameux qu'on appelle Demir capi, ou la Porte de Fer, comme vous sçavez mieux que moi. [...] (d'Arvieux, Mémoires VI: 134)

Les termes employés par ses deux correspondants construisent ainsi en filigrane le portrait du chevalier d'Arvieux: un voyageur éclairé, curieux de tout et possédant une érudition polymorphe, découlant d'une longue expérience viatique et interculturelle. En effet, ces quelques détails recueillis au fil des pages de la correspondance révèlent l'accumulation progressive d'un savoir hétérogène, qui conjugue naturellement la "connaissance parfaite» de la botanique et de la géographie avec celle des coutumes des Chrétiens d'Orient - en l'occurrence à un niveau plus mondain, celui de la réception des invités de marque. Qui plus est, l'excellente connaissance que le chevalier d'Arvieux a de l'Orient s'en trouve pleinement confirmée, non sans une pointe d'autodérision dans le cas de Casmon.

\section{La lettre érudite: entre mondanité et interculturalité}

Cependant, l'érudition des voyageurs d'Orient a un caractère «nomade», à l'image de leurs parcours accidentés, aventureux, périlleux, voire mortels. De même, leurs lettres, écrites bien souvent à la hâte - comme François Picquet ne manque pas de le souligner à plusieurs reprises - se font le reflet mouvant des hasards de la route, des rencontres de fortune, des informations recueillies dans des conditions qui feraient perdre toute envie de disserter à des gens moins hardis qu' eux. Ce type de savoir est moins une érudition spécialisée qu'une connaissance encyclopédique héritée de l'humanisme de la Renaissance et de la pratique du voyage telle qu'elle se développe au XvI ${ }^{\mathrm{e}}$ siècle ${ }^{15}$, mais qui, à l'âge classique, se tourne de plus en plus vers une exploration profane du monde et des cultures. La forme épistolaire s'imprègne

13. Ville de l'actuelle Turquie ayant appartenu à l'Arménie et à la Géorgie.

14. Ici au sens de plantes médicinales.

15. Sur les rapports ambigus qui lient à l'époque curiositas et studiositas, voir Gomez-Géraud (2013). 
ainsi d'un certain dynamisme propre au voyage et à l'échange qui se traduit par des pratiques d'écriture et des thématiques spécifiques.

Dans ce contexte, le non-dit apparaît comme particulièrement révélateur non seulement d'une certaine complicité des correspondants, mais aussi de tout un savoir partagé qui reste souvent implicite sous peine de devenir redondant. Les lettres de Picquet et de Casmon témoignent, à travers des tonalités qui leur sont propres, d'un savoir et d'un goût de l'Ailleurs que seuls des voyageurs ayant sillonné l'Orient sont à même de comprendre intimement. D'où toute une série de sous-entendus, de clins d'œil disséminés à travers la correspondance, qui apparaissent comme autant de marques de reconnaissance et de confirmation d'une érudition qui leur est caractéristique.

Si certains détails jugés superflus ne sont pas repris dans les lettres adressées au curieux d'Arvieux, comme nous l'avons vu, d'autres parsèment la correspondance et sont dignes d'être mentionnés:

A tout autre qu’à vous je ferois le détail de ces festins, mais je suis sûr que vous en sçavez plus que je ne pourrois vous en dire. Voici cependant quelque chose de si singulier, qu'il faut que je vous le dise. Notre compagnie fut fort surprise de voir deux grands flambeaux allumés sur la table en plein midi. [...] En voilà assez peut-être pour vous ennuyer ou pour vous divertir. (d'Arvieux, Mémoires VI: 99-100)

La connivence, évoquée plus haut, entre connaisseurs de l'Orient, qui pousse souvent les témoins à taire plutôt qu'à dire, se double, ici comme ailleurs, d'une petite pointe de fierté car ils ont quelque chose de «singulier» à raconter. L'évocation des deux grands flambeaux placés au centre d'une grande table et allumés en plein midi, mentionnés également pas Casmon dans l'une de ses lettres, témoigne de cette chasse aux "singularités» dont aussi bien les voyageurs que leur public de curieux sont friands à l'époque ${ }^{16}$. La surprise de l'évêque et de ses compagnons de route, qui explique la mention des deux flambeaux, laisse cependant le destinataire de la lettre qu'est d'Arvieux - et, par ricochet, le public curieux - sur sa faim, la bizarrerie de la situation ne recevant aucun éclaircissement supplémentaire. Tout aussi surprenante est la dernière phrase du passage: "En voilà assez peut-être pour vous ennuyer ou pour vous divertir", le couple pascalien ennui/divertissement faisant étrangement irruption lors d'une «situation d'altérité» que l'évêque se plaît à entretenir (Pirotte 2006: 25). Il est difficile de dire si l'auteur, qui excelle dans l'art du clin d'œil, entend ici instruire son lecteur, ou, au contraire, s'il présente son propre savoir sur le mode du divertissement pour ne pas l'ennuyer.

Quoi qu'il en soit, c'est bien à un savoir le plus souvent implicite, partagé entre initiés, que viennent se greffer les éléments nouveaux amenés par l'expérience viatique. Voici quelques remarques de l'aumônier Casmon faites à l'occasion de son passage et de celui de l'évêque en Perse:

[...] nous commençâmes à respirer quand nous entrâmes dans les Etats de Perse, en passant au pied du Mont Ararat. Les Armeniens l'appellent Massis, c'est la borne des deux Empires. Les Devots l'appellent la Montagne Sainte, parce qu'ils la regardent par tradition comme la Montagne sur laquelle s'arrêta l'Arche après le Déluge. Ceux qui en font sortir les quatre sources de quatre fameuses rivieres, l'Euphrate, le Tigre, le Faze et l'Araxe se trompent très-fort: s'ils avoient été sur les lieux ils en conviendroient, à moins qu'ils ne veulent fortifier par-là leur opinion aussi mal fondée, que le Paradis Terrestre étoit sur cette Montagne. (d'Arvieux, Mémoires VI : 135)

16. Cette chasse aux singularités n'est d'ailleurs pas sans rappeler celle de leurs devanciers du Xvi ${ }^{\mathrm{e}}$ siècle, parmi lesquels figurent des voyageurs comme André Thevet ou Pierre Belon. 
Ce passage est un exemple paradigmatique de la correspondance érudite qu'entretiennent les voyageurs en Orient. Ancrée dans une géographie des lieux de mémoire, l'écriture procède par accumulation aussi bien des références linguistiques et savantes que des points de vue (les Arméniens, les dévots, "ceux qui»), afin de mieux relativiser un savoir livresque et, somme toute, «mythologique». Dans un esprit polémique, l'auteur se place dans la position du témoin à même d'opposer l'observation directe - l'autopsie $^{17}$ - de celui qui a véritablement "été sur les lieux» aux opinions mal fondées de ceux qui, en se «trompant très fort», situent le Paradis Terrestre sur le mont Ararat ${ }^{18}$. Le démenti érudit cherche ici à subvertir l'érudition de cabinet selon une confrontation devenue de plus en plus fréquente, grâce à la multiplication des voyages, entre le monde et la bibliothèque, entre l'expérience directe et un savoir caduc, mis en échec par le nouvel esprit critique et expérimental qui s'exprime dans la correspondance des voyageurs. Dès lors, on voit se développer une forme d'écriture sinueuse, lacunaire, procédant par accumulations et révisions qui, loin d'être systématiques, forment comme des entrelacs de savoirs disséminés çà et là à travers la correspondance.

Quant à la thématique des lettres, elle recoupe souvent celle du genre auquel elles s'apparentent, à savoir la relation de voyage. C'est ainsi que l'abondance de ces bribes de savoir, qu'il soit historique, géographique, linguistique, culturel ou autre, suit la progression du voyageur à travers de nouvelles contrées. Pour commencer, le lecteur se familiarise avec «les disgraces d'un très-fâcheux voyage» (d'Arvieux, Mémoires VI : 107) : les obstacles et les périls naturels (traversée périlleuse des montagnes, froid et chaleur extrêmes, intempéries), mais aussi les souffrances et les douleurs physiques (maladies plus ou moins sévères ou "incommodités" de toutes sortes). Les deux correspondants les présentent chacun à sa manière. L'évêque s'y réfère parfois, mais sans véritablement s'y attarder, car, dit-il, "de vous entretenir de nos souffrances, ce seroit un excès de délicatesse ${ }^{19}$ " (d'Arvieux, Mémoires VI : 124), cette discrétion et cette pudeur devant une forme d'expression trop directe relevant d'une rhétorique classique de la litote. Quant à l'aumônier Casmon, il s'étend longuement sur les souffrances corporelles causées par le voyage ${ }^{20}$, tout en profitant de l'occasion pour faire l'éloge de l'évêque:

Il nous soûtient par son exemple; il supporte le froid le plus cuisant, et les plus brûlantes ardeurs du soleil, avec un visage serain, guai et content; quoiqu'il ait déjà perdu la peau du visage, et qu'il ait passé huit journées entieres moüillé jusqu’à la peau, jamais nous n’avons remarqué la moindre alteration sur son visage. (d'Arvieux, Mémoires VI : 106)

Moins héroïque que son supérieur, Casmon n’hésite pas à mettre en avant les moindres «incommodités» qui le poussent à prendre des mesures exceptionnelles: "[...] mais je dois vous dire qu'il n'y a rien de si incommode que d'être porté sur un chameau; je ne pus le souffrir, et je pris le parti d'aller à pied [...]» (d'Arvieux, Mémoires VI: 106). Ici, le savoir viatique met en lumière la difficulté d'apprendre à faire comme l'Autre, les pratiques orientales de la mobilité s'avérant une véritable épreuve qui débouche sur une initiation échouée. D'ailleurs, l'Autre lui-même représente souvent un obstacle, voire un danger. Les deux correspondants du chevalier d'Arvieux s'attardent ainsi sur l' «inhumanité» et la «friponnerie» des deux guides, Hage Cheleby et son fils Abdala, sur l'éternel problème des douanes

17. Sur la question de l'autopsie, voir Hartog (1980).

18. Sur l'évolution des représentations mentales des temps historiques à l'âge classique, et en particulier sur les débats concernant la localisation du Paradis terrestre, voir Poulouin (1998).

19. Ici dans le sens négatif de mollesse, voire d'affectation.

20. Sur l'importance du vécu corporel pour la connaissance de l'Ailleurs, voir Antoine (2014). 
et des péages de l'Empire ottoman, ainsi que sur leur crainte permanente des Arabes pillards, véritable topos des récits de voyage de l'époque.

Enfin, les dernières lettres de François Picquet brossent un tableau de la Perse safavide ${ }^{21}$ et de la ville d'Ispahan tout aussi éclectique et fragmentaire que celui de leur parcours, d'autant plus que quelques-unes d'entre elles ont été perdues. Comme dans la plupart des récits de voyage qui lui sont consacrés (par Chardin, Tavernier, etc.), l'image de la Perse proposée par François Picquet est empreinte d'ambivalence, étant construite par la perception d'un ecclésiastique devenu en quelque sorte ambassadeur malgré lui. Le lecteur apprend ainsi des détails intéressants au sujet du statut des ambassadeurs étrangers en Perse: malgré une autorité qui «surpasse infiniment celle de tous les Ambassadeurs en Europe et ailleurs" (d'Arvieux, Mémoires VI: 128) et dont l'évêque se prévaut plus d'une fois pour secourir des familles de Chrétiens, celle-ci entraîne également des obligations auxquelles il ne pourra pas se soustraire. Il lui faudra assumer ses fonctions d'ambassadeur dès son entrée en Perse et agir en conséquence, ce qui implique des dépenses et un train de vie dont l'évêque rendra compte de la sorte:

Cela m’oblige à faire travailler à un équipage qui vous surprendroit si vous le voyiez [...] vous ne me connoîtriez plus; je ne suis plus ce pauvre Evêque habillé d'une soutanne noire ou violette; on ne parle plus chez moi que de brocards, de toile d'or et d'argent: on veut que j'aye des chevaux de main, des housses en broderie; c'est un bonheur pour moi que les carosses ne sont pas à la mode [...] ma consolation est de sortir le plûtôt que je pourrai, et bientôt de cet état violent où je me trouve [...]. (d'Arvieux, Mémoires VI : 139-141)

Cette lamentation sur son triste sort n'est cependant pas dépourvue d'une certaine coquetterie mondaine, signe que, malgré la difficulté à concilier des fonctions à première vue incompatibles, le contact prolongé avec l'altérité provoque une certaine capacité d'adaptation. C’est toujours «malgré [s]a répugnance» (d'Arvieux, Mémoires VI : 142) qu'il racontera une série d'anecdotes viatiques savantes parfois bien savoureuses, comme celle de la rencontre entre le Chah de Perse et le roi des Yuzbeks ${ }^{22}$ et des difficultés de cérémonial ayant conduit à son report ou l'évocation d'autres fêtes ou réjouissances publiques. De même, il ne pourra s'empêcher d'émettre des jugements esthétiques favorables à l'égard de l'architecture persane, allant jusqu'à considérer certains de leurs bâtiments publics "plus beaux et plus superbes que nos bâtimens d'Europe» (d'Arvieux, Mémoires VI: 141). Ces jugements vont même jusqu'à se conjuguer avec d'autres d'ordre anthropologique et comparatif, voire relativisant, du type: "ils ne laissent pas d'être incommodes pour nos usages, mais chaque Nation a les siens» (d'Arvieux, Mémoires VI : 142). Bref, voilà un tableau bariolé de la Perse safavide qui témoigne d'un esprit tout à la fois érudit, missionnaire et mondain.

À une époque où l'Ailleurs, loin d'être banalisé, invite à l'exploration et à la réflexion, les lettres échangées par les voyageurs en Orient au sein de leurs réseaux interpersonnels et savants témoignent de la fraîcheur nomade de la découverte. La correspondance entretenue par le chevalier d'Arvieux avec l'évêque-ambassadeur François Picquet et son aumônier Casmon illustre ainsi à merveille les caractéristiques particulières de la lettre érudite en contexte viatique et interculturel. Située à mi-chemin entre le monde et la bibliothèque, entre un savoir encyclopédique et un savoir plus proprement viatique et anthropologique, la correspondance des voyageurs reproduit, avec toutes leurs aspérités, les aléas de

21. Pour plus de détails sur les représentations de la Perse à l'âge classique, ainsi que sur les rapports souvent problématiques entre les voyageurs européens et les Persans, voir Tinguely (2018) et Jacquin (2010).

22. Ouzbeks. 
la route. Relevant à la fois d'une culture de la mobilité, de la curiosité et de l'érudition, ces échanges épistolaires apparaissent comme une contribution indéniable à l'avancement du savoir au sein de la République des Lettres orientalistes de l'âge classique.

\section{Références bibliographiques}

Références primaires

Antelmy, C. L. O. 1732. La vie de messire François Picquet. Paris: Chez la Veuve Merge.

Arvieux, L. d'. 1735. Mémoires du chevalier d'Arvieux, envoyé extraordinaire du Roi à la Porte, Consul d'Alep, d'Alger, de Tripoli et autres Échelles du Levant. Édition préparée par J.-B. Labat. Paris: Charles-Jean-Baptiste Delespine.

Tavernier, J.-B. 1676. Les six voyages [...] en Turquie, en Perse et aux Indes. Paris: Gervais Clouzier et Claude Barbin.

Références secondaires

Antoine, P., dir. 2014. Viatica 1: Le corps du voyageur. http://viatica.univ-bpclermont.fr/le-corps-du-voyageur. Article consulté pour la dernière fois le 26 août 2018.

Antoine, P. et V. Pârlea, dir. 2018. Voyage et intimité. Paris: Classiques Garnier.

Beaurepaire, P.-Y. et H. Hermant, dir. 2012. Entrer en communication: de l'âge classique aux Lumières. Paris: Classiques Garnier.

Häseler, J. et A. McKenna. 2006. «Introduction: De la lettre à la correspondance et du réseau à l'espace social». Dans Réseaux de correspondance à l'âge classique (XVI -XVIII siècle), sous la direction de P.-Y. Beaurepaire, J. Häseler et A. McKenna. Saint-Étienne: Publications de l'Université de Saint-Étienne: 7-16.

Dew, N. 2009. Orientalism in Louis XIV's France. Oxford: Oxford University Press.

El-Mudarris, H. I. et O. Salmon, dir. 2009. Le consulat de France à Alep au XVII siècle: Journal de Louis Gédoyn, Vie de François Piquet, Mémoires de Laurent d'Arvieux. Alep: Ray Publishing and Science.

Girard, A. 2015. «Introduction». Dix-septième siècle 268 (3) : 385-392.

Gomez-Géraud, M.-C. 2013. «La curiosité, qualité du voyageur? Succincte enquête sur la littérature viatique du XVI ${ }^{\mathrm{e}}$ siècle». Dans Camenae 15: 1-10, http://lettres.sorbonne-universite.fr/IMG/pdf/9-Gomez-Geraud. pdf. Article consulté pour la dernière fois le 25 août 2019).

Haffemayer, S. 2008. "Transferts culturels dans la presse européenne au XviI ${ }^{\mathrm{e}}$ siècle». Le Temps des médias 11: $25-43$.

Hartog, F. 1980. Le miroir d'Hérodote: Essai sur la représentation de l'autre. Paris: Gallimard.

Heyberger, B. 2015. «L'Orient et l'Islam dans l'érudition européenne du XvII ${ }^{\mathrm{e}}$ siècle». Dix-septième siècle 268 (3) : 495-508.

Hossain, M. 1990. «The Chevalier d'Arvieux and Le bourgeois gentilhomme». Seventeenth-Century French Studies $12: 76-88$.

Hossain, M. 1997. "The Lebaudy manuscript of the Mémoires of the chevalier d'Arvieux : the question of authenticity revisited ». Revue d'Histoire Maghrébine 85-86: 103-117.

Jacquin, F. 2010. Le voyage en Perse au XVII siècle. Paris: Éditions Belin.

Le Huenen, R. 1990. "Qu'est-ce qu'un récit de voyage?». Littérales 7: 11-27.

Lewis, W. H. 1962. Levantine Adventurer: The travels and missions of the Chevalier d'Arvieux (1653-1697). Londres: Andre Deutsch.

Moureau, F. 2005. Le théatre des voyages: Une scénographie de l'Âge classique. Paris: Presses de l'Université Paris-Sorbonne.

Pârlea, V. 2015. «Un Franc parmy les Arabes»: Parcours oriental et découverte de l'Autre chez le chevalier d'Arvieux. Grenoble: ELLUG. 
Pirotte, J. 2006. «Histoire et altérité. Rencontre, rejet, métissage». Dans De l'Orient à l'Occident et retour: Perceptions et représentations de l'Autre dans la littérature et les guides de voyage, sous la direction de P. Servais. Louvain-la-Neuve: Academia Bruylant: 21-45.

Poulouin, C. 1998. Le temps des origines: L'Éden, le Déluge et «les temps reculés». De Pascal à L’Encyclopédie. Paris: Champion.

Salmon, O. 2011. Alep dans la littérature de voyage européenne pendant la période ottomane (1516-1918), t. I. Alep: Dar al-Mudarris \& Dar Mardin.

Schapira, N. 2003. Un professionnel des lettres au XVIt siècle: Valentin Conrart: une histoire sociale. Seyssel: Champ Vallon.

Tinguely, F., dir. 2018. Dix-septième siècle 278 (1) : 111-122.

Viala, A. 1985. Naissance de l'écrivain. Sociologie de la littérature à l'âge classique. Paris: Éditions de Minuit.

Wolfzettel, F. 1996. Le discours du voyageur: Pour une histoire littéraire du récit de voyage en France, du Moyen Âge au XVIII siècle. Paris: Presses Universitaires de France.

Zeiller, J. 1943. «Un précurseur: François Picquet, consul de Louis XIV en Alep et évêque de Babylone». Journal des Savants $4:$ 145-157. 